\title{
DERIVATIVES OF THE CONFORMAL CAPACITY OF EXTREMAL RINGS
}

\author{
GLEN D. ANDERSON
}

1. Introduction. In this paper we illustrate a method for deriving information concerning the derivatives of the conformal capacity of certain extremal rings in $n$-space. For fixed $n \geqq 3$ and $1 \leqq p \leqq n-1$, these rings are denoted by $R_{n, p}$ where, for $0<t<1$,

$$
R_{n, p}(t)=B^{n} \backslash \overline{B^{p}(t)} ;
$$

that is, $R_{n, p}$ is the ring consisting of the open unit ball in euclidean $n$-space minus the closed concentric $p$-dimensional ball of radius $t$. Usually we identify $R^{p}$ with the subset $\left\{\left(x_{1}, \ldots, x_{p}, 0, \ldots, 0\right): x_{j}\right.$ real, $\left.1 \leqq j \leqq p\right\}$ of $R^{n}$.

The conformal capacity of $R=R_{n, p}(t)$ is defined, as usual, to be

$$
\mu=\mu_{n, p}(t)=\operatorname{cap} R_{n, p}(t)=\inf _{u} \int_{R_{n, p}}|\nabla u|^{n} d \omega,
$$

where $u \in C^{1}(R), u=0$ on $\overline{B^{p}(t)}$, and $u=1$ on $S^{n-1}$.

The modulus of $R$ is defined by

$$
\bmod R=\left(\sigma_{n-1} / \mu\right)^{1 /(n-1)}, \quad \sigma_{n-1}=m_{n-1}\left(S^{n-1}\right) .
$$

It is well known [1] that $\bmod R_{2,1}(t)=\pi K^{\prime} /(4 K)$, so that

where

$$
\mu_{2,1}(t)=\operatorname{cap} R_{2,1}(t)=2 \pi / \bmod R_{2,1}(t)=8 K / K^{\prime},
$$

$$
K=K(t)=\int_{0}^{1}\left[\left(1-x^{2}\right)\left(1-t^{2} x^{2}\right)\right]^{-1 / 2} d x, \quad K^{\prime}=K\left(t^{\prime}\right), \quad t^{\prime}=\left(1-t^{2}\right)^{1 / 2} .
$$

If one can show that among all rings with a certain geometric property a particular ring is extremal, that is, has the maximum modulus, then this fact can be used to determine distortion properties for quasiconformal mappings (cf. [15]). The rings $R_{n, p}$ have the following extremal property (cf. Theorems 4 and 5 in [1]). Let $R$ be any ring in $R^{n}$ consisting of the unit ball minus a continuum $C$, and suppose the projection of $C$ onto some $p$-dimensional linear subspace of $R^{n}$ has $p$-dimensional measure at least $m_{p} B^{p}(t), 0<t<1$. Then $\bmod R \leqq \bmod R_{n, p}(t)$.

AMS (MOS) subject classification (1980): Primary 30C60. Secondary 30C70, 31B35, 33A25, $35 \mathrm{~J} 65$. 
It is well known [15] that if a ring $R$ has nondegenerate boundary components then there is a unique admissible extremal function $u$ for the variational problem (1). By standard techniques of the calculus of variations it may be shown that the Euler equation for this extremal problem in $R$ is the quasilinear (degenerate) elliptic partial differential equation (cf. [15], [24])

$$
\operatorname{div}\left(|\nabla u|^{n-2} \nabla u\right)=0
$$

of which the extremal function $u$ is a weak solution.

Since each of the elliptic integrals $K$ and $K^{\prime}$ satisfies the ordinary differential equation $t\left(1-t^{2}\right) u^{\prime \prime}+\left(1-3 t^{2}\right) u^{\prime}-t u=0$ ([10, Formula 118.02], [9, p. 21]), where ' denotes differentiation with respect to $t$, it follows from the theory of ordinary differential equations [21] that $w=\mu_{2,1}(t)$ satisfies the differential equation

$$
S_{w}(t)=\frac{1}{2}\left(\left(1+t^{2}\right) /\left(t-t^{3}\right)\right)^{2},
$$

where $S_{w}$ denotes the Schwarzian derivative $\left(w^{\prime \prime} / w^{\prime}\right)^{\prime}-\left(w^{\prime \prime} / w^{\prime}\right)^{2} / 2$. The differential equation (5) was originally derived before 1890 (before the introduction of the Grötzsch ring) without the benefit of the Schwarzian derivative and using only the properties of elliptic integrals (cf. [13]).

Since an exact formula for the capacity is not known when $n \geqq 3$, it is much more difficult to obtain information about the derivatives of $\mu_{n, p}$ for these $n$ than when $n=2$. However, in this paper we illustrate a variational technique by means of which one may obtain integral representations for these derivatives, and then we estimate these integrals to find asymptotic limits for $\mu_{n, p}^{\prime}(t)$ as $t$ tends to 0 and to 1 and also to find an inequality satisfied by the second derivative $\mu_{n, p}^{\prime \prime}(t)$.

For the most part we shall follow the notation introduced in [2] and [4].

2. Acknowledgements. The author wishes to express his gratitude to $\mathbf{M} . \mathbf{M}$. Schiffer, who in private consultation contributed the ideas in Sections 6 and 7, and to T. Iwaniec for assistance with the argument in Section 3. The author also thanks D. H. Y. Yen and F. W. Gehring for conversations concerning this problem.

3. Smoothness of the extremal function. It has been shown by N. N. Ural'ceva [22] that if $u$ is a weak solution of (4) in a domain $\Omega \subset R^{n}$ with $\partial \Omega \in C^{\infty}$, then $u \in C^{1, \eta}(\bar{\Omega})$; that is, $u \in C^{1}(\bar{\Omega})$ and $\nabla u$ is Hölder continuous with exponent $\eta>0$ in $\bar{\Omega}$. An example of B. Bojarski and T. Iwaniec shows that this is a best possible result as to smoothness [7].

In the present case this degree of smoothness up to $S^{n-1}$ for the extremal function $u$ for $R_{n, p}$ permits the calculations in Sections 6 and 7 to be carried out. It is easy to verify directly that the function $\hat{u}(P)=2-u\left(P /|P|^{2}\right)$ is a solution of (4) in the ring $\hat{R}$ which is the reflection of $R_{n, p}$ in $S^{n-1}$ and that $\hat{u}$ has the boundary value $\hat{u}=1$ on $S^{n-1}$. 
Since $|\nabla u|>0$ on $S^{n-1}$ by Lemmas 5 and 6 below, we may appeal to Nirenberg [19] and Morrey [18] to conclude that $u$ is even real analytic in $R_{n, p}$ near $S^{n-1}$. Likewise $\hat{u}$ is real analytic outside of $R_{n, p}$ near $S^{n-1}$. Since $u=\hat{u}$ and $\nabla u=\nabla \hat{u}$ on $S^{n-1}$, the sphere $S^{n-1}$ is a removable singular set (as in the plane harmonic case). Hence $u$ is real analytic in a neighborhood of $S^{n-1}$. Thus the calculations needed to justify the formula (54) for the second derivative $\mu$ " are valid.

4. Symmetry of the extremal function. We want to show that for each $n \geqq 3$, $1 \leqq p \leqq n-1,0<t<1$, there is a two-dimensional subset of $R_{n, p}$ such that all the values of the extremal function $u$ in $R_{n, p}$ may be realized by appropriately rotating and reflecting the values of $u$ taken on in this plane set.

Lemma 1. If $u$ is the extremal function for cap $R_{n, p}(t), n \geqq 3,1 \leqq p \leqq n-1$, $0<t<1$, then $u$ has the same symmetries as $R_{n, p}$.

Proof. First, suppose $p=1$. It is convenient to use cylindrical coordinates $(x, y, e)$ in $R^{n}$, where $x=x_{1}, y$ is the length of the vector $\left(x_{2}, \ldots, x_{n}\right)$, and $e \in S^{n-2}$ (cf. [2], p. 4). If $u=u(x, y, e)$, then the function $u^{*}=u^{*}(x, y)$ obtained by averaging the values of $u(x, y, e)$ over $S^{n-2}$ is admissible for cap $R_{n, 1}(t)$ and symmetric about the $x$-axis. Hölder's inequality implies that

$$
\left|\nabla u^{*}\right| \leqq\left(\frac{1}{\sigma_{n-2}} \int_{S^{n-2}}|\nabla u(x, y, e)|^{n} d m_{n-2}(e)\right)^{1 / n} .
$$

By multiplying both sides of this inequality by $\sigma_{n-2}^{1 / n}$, raising both sides to the power $n$, and integrating over the plane set

$$
B_{+}^{2}=\left\{(x, y): x^{2}+y^{2}<1, y \geqq 0\right\}
$$

we may show that the integral of $\left|\nabla u^{*}\right|^{n}$ over $R_{n, 1}(t)$ is at most cap $R_{n, 1}(t)$. By uniqueness of the extremal function we conclude that $u^{*}=u$, so that $u$ must be already symmetric with respect to the $x$-axis.

We may show further that the extremal function $u$ must be symmetric with respect to the hyperplane $x=0$ in $R^{n}$. For let $u^{*}(x, y)=(u(x, y)+u(-x, y)) / 2$ in $B_{+}^{2}$ and extend $u^{*}$ to all of $R_{n, 1}$ by symmetry. Then, by an elementary inequality,

$$
\left|\nabla u^{*}(x, y)\right| \leqq\left(\frac{1}{2}\left(|\nabla u(x, y)|^{n}+|\nabla u(-x, y)|^{n}\right)\right)^{1 / n}
$$

in $B_{+}^{2}$. It is now easy to show that the integral of $\left|\nabla u^{*}\right|^{n}$ over $R_{n, 1}$ does not exceed cap $R_{n, 1}$, and we may conclude that $u$ has the desired symmetry.

Next, if $p=n-1$, we may achieve the analogous result concerning symmetry about the $y$-axis and the hyperplane $y=0$ in $R^{n}$ by taking $y=x_{n}$ and $x$ to be the length of the vector $\left(x_{1}, \ldots, x_{n-1}\right)$ in the above argument.

Finally, if $2 \leqq p \leqq n-2$, we may use the procedure suggested by J. Sarvas in $\left[2\right.$, p. 12] to represent any point $\left(x_{1}, \ldots, x_{n}\right) \in R^{n}$ in coordinates $\left(x, y, e_{1}, e_{2}\right)$, where $x$ and $y$ are the lengths of the vectors $\left(x_{1}, \ldots, x_{p}\right)$ and $\left(x_{p+1}, \ldots, x_{n}\right)$, respectively, and 
$e_{1}$ and $e_{2}$ belong to $(p-1)$ - and $(n-p-1)$-dimensional spheres $S_{1}$ and $S_{2}$, respectively. For any $e_{1} \in S_{1}$ and $e_{2} \in S_{2}$ we may think of $R_{n, p}(t)$ as being obtained from the two-dimensional set

$$
Q\left(e_{1}, e_{2} ; t\right)=\left\{x e_{1}+y e_{2}: x^{2}+y^{2}<1, x \geqq 0, y \geqq 0\right\} \backslash\left\{x e_{1}: 0 \leqq x \leqq t\right\}
$$

by rotation around $S_{1}$ and $S_{2}$. If $u=u\left(x, y, e_{1}, e_{2}\right)$ is extremal for cap $R_{n, p}$, then the function $u^{*}(x, y)$ obtained by averaging the values of $u\left(x, y, e_{1}, e_{2}\right)$ over $S_{1} \times S_{2}$ is admissible for cap $R_{n, p}$ and symmetric with respect to $S_{1}$ and $S_{2}$. By the same argument used above we may then show that $u^{*}=u$ in $R_{n, p}$, so that $u$ must have the same rotational symmetries as the ring $R_{n, p}$.

Remark. Lemma 1 implies that the Euler equation (4) for cap $R_{n, p}$ may always be reduced to a partial differential equation in two independent variables in a set $\mathscr{D}=\left\{(x, y): x^{2}+y^{2}<1, x>0, y>0\right\}$.

5. A coordinate form of the Euler equation. For $k=t^{2}, z=x+i y$, and $\zeta=\alpha+i \beta$, let $z=f(\zeta)=\sqrt{k} \operatorname{sn}(\zeta, k)$ be the plane conformal mapping of the rectangle $\{\zeta: 0<\alpha<K$, $\left.0<\beta<K^{\prime} / 2\right\}$ onto the open first quadrant $\mathscr{D}$ of the unit disk in the $z$-plane, with boundary values $0, t, 1, i$ at $0, K, K+i K^{\prime} / 2, i K^{\prime} / 2$, respectively. Here $s n$ denotes the Jacobian elliptic sine function ([9, Chapter 5], [10, Formula 119.01]) and $K$ and $K^{\prime}$ are the elliptic integrals in (3). It will be particularly convenient to write the Euler equation (4) using the variables $\alpha$ and $\beta$ inherited from this conformal mapping. The coordinate curves $\alpha=$ constant and $\beta=$ constant in $\mathscr{D}$ are known as bicircular quartics [9].

When working with the ring $R_{n, 1}$ it is appropriate to employ a cylindrical coordinate system having the $x_{1}$-axis as the axis of symmetry. The relationship between rectangular coordinates $\left(x_{1}, \ldots, x_{n}\right)$ and such cylindrical coordinates $\left(x, y, \theta_{1}, \ldots\right.$, $\left.\theta_{n-2}\right)$ in $R^{n}$ may be written in detail as

$$
\begin{aligned}
& x_{1}=x, \\
& x_{2}=y \sin \theta_{1} \ldots \sin \theta_{n-3} \sin \theta_{n-2}, \\
& \quad \ldots \\
& x_{j}=y \sin \theta_{1} \ldots \sin \theta_{n-j} \cos \theta_{n-j+1}, \\
& \quad \ldots \\
& x_{n}=y \cos \theta_{1}
\end{aligned}
$$

(cf. [11], p. 237). In our case, for the ring $R_{n, 1}$, we want to take $x=\operatorname{Re} f(\zeta)$ and $y=\operatorname{Im} f(\zeta)$, where $\zeta=\alpha+i \beta$.

We may write the gradient and divergence operators in the curvilinear coordinate system $\left(\alpha, \beta, \theta_{1}, \ldots, \theta_{n-2}\right)$ as

$$
\nabla \varphi=h_{\alpha}^{-1} \varphi_{\alpha} e_{\alpha}+h_{\beta}^{-1} \varphi_{\beta} e_{\beta}+\sum_{j=1}^{n-2} h_{\theta_{j}}^{-1} \varphi_{\theta_{j}} e_{\theta_{j}}
$$


and

$$
\operatorname{div} V=H^{-1}\left[\frac{\partial}{\partial \alpha}\left(V_{\alpha} H / h_{\alpha}\right)+\frac{\partial}{\partial \beta}\left(V_{\beta} H / h_{\beta}\right)+\sum_{j=1}^{n-2} \frac{\partial}{\partial \theta_{j}}\left(V_{\theta_{j}} H / h_{\theta_{j}}\right)\right],
$$

where $h_{\alpha}, h_{\beta}, h_{\theta_{j}}, j=1, \ldots, n-2$ are the scale factors and $H$ is their product (cf. [6]). In the present case, rotational symmetry implies that all partials of the extremal function $u$ in the directions $e_{\theta_{j}}$ are identically zero, so that we can drop all but the first two terms in (6) when $\varphi=u$ or when $\varphi$ has the same symmetries as $u$.

Next, $h_{\alpha}=h_{\beta}=\left|f^{\prime}\right|$, while we may show that $H=y^{n-2}\left|f^{\prime}\right|^{2} \Phi$, where $\Phi$ is independent of $\alpha$ and $\beta$. Hence if we put

$$
V=|\nabla u|^{n-2} \nabla u=\left|f^{\prime}\right|^{1-n}\left(u_{\alpha}^{2}+u_{\beta}^{2}\right)^{(n-2) / 2}\left(u_{\alpha} e_{\alpha}+u_{\beta} e_{\beta}\right)
$$

in (7) the Euler equation (4) becomes

$$
\begin{aligned}
Q_{n} u= & \operatorname{div}\left(|\nabla u|^{n-2} \nabla u\right) \\
= & y^{2-n}\left|f^{\prime}\right|^{-2}\left[\frac{\partial}{\partial \alpha}\left(\left(y /\left|f^{\prime}\right|\right)^{n-2}\left(u_{\alpha}^{2}+u_{\beta}^{2}\right)^{(n-2) / 2} u_{\alpha}\right)\right. \\
& \left.+\frac{\partial}{\partial \beta}\left(\left(y /\left|f^{\prime}\right|\right)^{n-2}\left(u_{\alpha}^{2}+u_{\beta}^{2}\right)^{(n-2) / 2} u_{\beta}\right)\right]=0 .
\end{aligned}
$$

When we carry out the computations on the right in (8) and simplify and then use the obvious modification $(1 / g)^{\prime}=-g^{-3}\left(g^{2}\right)^{\prime} / 2$, where $g=\left|f^{\prime}\right| / y$ and ' is $\partial / \partial \alpha$ or $\partial / \partial \beta$, we arrive at

Lemma 2. The Euler equation for the extremal problem (1) for $R_{n, 1}$ may be written as

$$
\begin{gathered}
(n-2)\left(u_{\alpha}^{2} u_{\alpha \alpha}+2 u_{\alpha} u_{\beta} u_{\alpha \beta}+u_{\beta}^{2} u_{\beta \beta}\right)+\left(u_{\alpha}^{2}+u_{\beta}^{2}\right)\left(u_{\alpha \alpha}+u_{\beta \beta}\right) \\
-\frac{1}{2}(n-2)\left(u_{\alpha}^{2}+u_{\beta}^{2}\right)\left(y /\left|f^{\prime}\right|\right)^{2}\left[\frac{\partial}{\partial \alpha}\left(\left(\left|f^{\prime}\right| / y\right)^{2}\right) u_{\alpha}+\frac{\partial}{\partial \beta}\left(\left(\left|f^{\prime}\right| / y\right)^{2}\right) u_{\beta}\right]=0
\end{gathered}
$$

in cylindrical coordinates $(\alpha, \beta, e)$ with $x$-axis as axis of symmetry, where $(\alpha, \beta)$ are bicircular quartic coordinates in $B_{+}^{2}$ and $e \in S^{n-2}$.

For the ring $R_{n, n-1}$ we need to interchange the roles of the $x$ - and $y$-axes in $R^{2}$, making the $x_{n}$-axis the axis of symmetry in $R^{n}$. If we carry out the above computations for this ring we obtain

Lemma 3. The Euler equation for the extremal problem (1) for $R_{n, n-1}$ may be written as

$$
\begin{gathered}
(n-2)\left(u_{\alpha}^{2} u_{\alpha \alpha}+2 u_{\alpha} u_{\beta} u_{\alpha \beta}+u_{\beta}^{2} u_{\beta \beta}\right)+\left(u_{\alpha}^{2}+u_{\beta}^{2}\right)\left(u_{\alpha \alpha}+u_{\beta \beta}\right) \\
-\frac{1}{2}(n-2)\left(u_{\alpha}^{2}+u_{\beta}^{2}\right)\left(x /\left|f^{\prime}\right|\right)^{2}\left[\frac{\partial}{\partial \alpha}\left(\left(\left|f^{\prime}\right| / x\right)^{2}\right) u_{\alpha}+\frac{\partial}{\partial \beta}\left(\left(\left|f^{\prime}\right| / x\right)^{2}\right) u_{\beta}\right]=0
\end{gathered}
$$


in cylindrical coordinates $(\alpha, \beta, e)$ with $y$-axis as axis of symmetry, where $(\alpha, \beta)$ are bicircular quartic coordinates in the right half of the unit disk in the $x y$-plane, and $e \in S^{n-2}$.

Remark. For later reference it is important to note here that $Q_{n} u=$ $\operatorname{div}\left(|\nabla u|^{n-2} \nabla u\right)$ is a positive (non-constant) multiple of the left side of the equations displayed in (9) and in (10).

Finally, for the rings $R_{n, p}, 2 \leqq p \leqq n-2$, we may modify the above procedures to obtain a coordinate system based on the ideas discussed in the proof of Lemma 1 above. The Euler equation (4) for $R_{n, p}$ in this coordinate system becomes

where

$$
\frac{\partial}{\partial \alpha}\left(\psi u_{\alpha}\right)+\frac{\partial}{\partial \beta}\left(\psi u_{\beta}\right)=0
$$

$$
\psi=\left(x /\left|f^{\prime}\right|\right)^{p-1}\left(y /\left|f^{\prime}\right|\right)^{n-p-1}\left(u_{\alpha}^{2}+u_{\beta}^{2}\right)^{(n-2) / 2} .
$$

However, rather than to make estimates based on this equation, we shall employ another technique in Lemma 6 of Section 9 to compare these rings with $R_{n, 1}$ and $R_{n, n-1}$.

6. A surface integral representing $\mu$. It has been shown by Gehring [16] and Ziemer [24] that if $u$ is extremal for a ring $R$, then for almost all $a \in[0,1]$,

$$
\int_{u^{-1}(a)}|\nabla u|^{n-1} d H^{n-1}=\operatorname{cap} R,
$$

where $H^{n-1}$ denotes the $(n-1)$-dimensional Hausdorff measure. We show here that an equation of type (11) holds for the rings $R_{n, p}$ when $a=1$, that is for $u^{-1}(1)$, which is $S^{n-1}$ by the maximum principle.

Theorem 1. For fixed $n \geqq 3$ and $1 \leqq p \leqq n-1$, let $R=R_{n, p}(t), 0<t<1$. If $u$ is extremal for $\mu=\operatorname{cap} R$, then

$$
\mu=\int_{S^{n-1}} u_{r}^{n-1} d \sigma .
$$

Proof. If $u$ is extremal for $\mu=\operatorname{cap} R$, then by Green's theorem (sometimes called integration by parts) $[12$, p. 69]

$$
\int_{R}|\nabla u|^{n} d \omega=\int_{\partial R} \nu \cdot\left(|\nabla u|^{n-2} \nabla u\right) u d \sigma-\int_{R} u \operatorname{div}\left(|\nabla u|^{n-2} \nabla u\right) d \omega,
$$

where $v$ represents the unit outer normal. But the last integral in (12) is 0 by the Euler equation (4) for cap $R$. Since $u=0$ on $\overline{B^{p}(t)}$ and $u=1$ on $S^{n-1}$, (12) reduces to

$$
\int_{R}|\nabla u|^{n} d \omega=\int_{S^{n-1}}|\nabla u|^{n-2} u_{r} d \sigma .
$$

Since $u$ is constant on $S^{n-1}$ the tangential components of $\nabla u$ are 0 there. Finally 
$u_{r} \geqq 0$ on $S^{n-1}$ since $0<u<1$ in $R$ [24, Corollary 3.11] and $u=1$ on $S^{n-1} \cdot{ }^{1}$ ) Thus $|\nabla u|=u_{r}$ on $S^{n-1}$ and the theorem is proved.

7. A surface integral representing $\mu^{\prime}$. We next prove

Theorem 2. For $n \geqq 3$ and $1 \leqq p \leqq n-1$, let $R=R_{n, p}(t), 0<t<1$. If $u$ is extremal for $\mu=\operatorname{cap} R$, then

$$
\mu^{\prime}=(n-1) t^{-1} \int_{S^{n-1}} u_{r}^{n} d \sigma,
$$

where' denotes differentiation with respect to $t$.

Proof. Deform $R$ into a ring $R^{*}=B^{n}(1+\varepsilon) \backslash \overline{B^{p}(t)}$ by means of a radial boundary deformation. Let $u$ and $u^{*}$ be extremal for $R$ and $R^{*}$, respectively. Take $u^{*}=$ $u+\varepsilon v+\left(\varepsilon^{2}\right)$ and use the binomial expansion to obtain

$$
\left|\nabla u^{*}\right|^{n}=|\nabla u|^{n}+n \varepsilon|\nabla u|^{n-2} \nabla u \cdot \nabla v+\left(\varepsilon^{2}\right) .
$$

Then for $\mu^{*}=\operatorname{cap} R^{*}$ we have

$$
\begin{aligned}
\mu^{*}= & \int_{R^{*}}|\nabla u|^{n} d \omega+n \varepsilon \int_{R^{*}}|\nabla u|^{n-2} \nabla u \cdot \nabla v d \omega+\left(\varepsilon^{2}\right) \\
= & \int_{R}|\nabla u|^{n} d \omega+\varepsilon \int_{S^{n-1}}|\nabla u|^{n} d \sigma+n \varepsilon \int_{R}|\nabla u|^{n-2} \nabla u \cdot \nabla v d \omega+\left(\varepsilon^{2}\right) \\
= & \int_{R}|\nabla u|^{n} d \omega+\varepsilon \int_{S^{n-1}}|\nabla u|^{n} d \sigma+n \varepsilon \int_{\partial R} v|\nabla u|^{n-2} u_{r} d \sigma \\
& -n \varepsilon \int_{R} v \operatorname{div}\left(|\nabla u|^{n-2} \nabla u\right) d \omega+\left(\varepsilon^{2}\right) \\
= & \int_{R}|\nabla u|^{n} d \omega+\varepsilon \int_{S^{n-1}}|\nabla u|^{n} d \sigma+n \varepsilon \int_{S^{n-1}} v|\nabla u|^{n-2} u_{r} d \sigma+\left(\varepsilon^{2}\right),
\end{aligned}
$$

where in the last two steps we have invoked Green's theorem and employed the Euler equation (4) as in the proof of Theorem 1.

If $P \in S^{n-1}(r)$, we write $P=(r, e)$ in spherical (polar) coordinates, where $r=|P|$ and $e \in S^{n-1}$. Then for $r=1+\varepsilon$ we have

$$
\begin{gathered}
1=u^{*}(1+\varepsilon, e)=u(1+\varepsilon, e)+\varepsilon v(1+\varepsilon, e)+\left(\varepsilon^{2}\right) \\
=u(1, e)+\varepsilon u_{r}(1, e)+\varepsilon v(1, e)+\left(\varepsilon^{2}\right),
\end{gathered}
$$

so that $v=-u_{r}$ when $r=1$. Then inserting this value of $v$ into the expression for $\mu^{*}$ obtained above and using the fact that $|\nabla u|=u_{r}$ on $S^{n-1}$, we arrive at

$$
\mu^{*}=\mu+\varepsilon(1-n) \int_{S^{n-1}} u_{r}^{n} d \sigma+\left(\varepsilon^{2}\right) .
$$

1) It has been shown by F. W. Gehring, in some unpublished notes, that if the components of the complement of $R$ are starshaped with respect to the origin then $u$ is radially nondecreasing in $R$. 
Finally, by conformal invariance of the capacity we have

$$
\mu^{*}=\mu(t /(1+\varepsilon))=\mu(t)-\varepsilon t \mu^{\prime}(t)+\left(\varepsilon^{2}\right),
$$

and comparison of the coefficient of $\varepsilon$ in (13) and (14) yields the theorem.

8. An application. It has been shown by Gehring [14] that for a ring $R=$ $=R_{n, p}(t)$ the function $\bmod R+\log t$ is monotone decreasing for $0<t<1$. We now show how this monotoneity follows from Theorems 1 and 2 above.

By Hölder's inequality,

$$
\int_{S^{n-1}} u_{r}^{n-1} d \sigma \leqq \sigma_{n-1}^{1 / n}\left(\int_{S^{n-1}} u_{r}^{n} d \sigma\right)^{(n-1) / n} .
$$

Raising both sides of this inequality to the power $n /(n-1)$ and substituting the expressions for $\mu$ and $\mu^{\prime}$ obtained in Theorems 1 and 2, we obtain

$$
\mu^{n /(n-1)} \leqq t(n-1)^{-1} \mu^{\prime} \sigma_{n-1}^{1 /(n-1)} .
$$

Rearranging, we may write this as

$$
-(n-1)^{-1} \sigma_{n-1}^{1 /(n-1)} \mu^{n /(1-n)} \mu^{\prime}+t^{-1} \leqq 0,
$$

which because of (2) reduces to the fact that $\bmod R+\log t$ has a nonpositive derivative with respect to $t, 0<t<1$.

9. Bounds for $u_{r}$ on $S^{n-1}$. In estimating $\mu^{\prime}$ it will be important to have upper and nontrivial lower bounds for the radial derivative of the extremal function on $S^{n-1}$.

Lemma 4. If $u$ is extremal for cap $R_{n, 1}(\sqrt{k})$, then on $S^{n-1}$

$$
(1+k)^{1 /(1-n)} A_{k}^{-1} \frac{1+k s^{2}}{1-k s^{2}} \leqq u_{r} \leqq \frac{2}{(1+k) K^{\prime}} \frac{1+k s^{2}}{1-k s^{2}},
$$

where

and

$$
A_{k}=\int_{0}^{K^{\prime} / 2}\left(\frac{d_{1}}{s_{1} c_{1}}\right)^{\frac{n-2}{n-1}} d \beta
$$

$$
\begin{aligned}
& s=s n(\alpha, k), c=c n(\alpha, k), d=d n(\alpha, k), \\
& s_{1}=\operatorname{sn}\left(\beta, k^{\prime}\right), \quad c_{1}=c n\left(\beta, k^{\prime}\right), d_{1}=d n\left(\beta, k^{\prime}\right) .
\end{aligned}
$$

Here $K^{\prime}=K\left(\left(1-t^{2}\right)^{1 / 2}\right)$ is as in (3) and sn, cn, dn are Jacobian elliptic functions.

Proof. First put $\varphi=\varphi(\beta)=2 \beta / K^{\prime}$ in the quarter disk $\mathscr{D}=\left\{(x, y): x^{2}+y^{2}<1\right.$, $x>0, y>0$, where $x$ and $y$ are the real and imaginary parts of $f(\zeta), \zeta=\alpha+i \beta$ (see Section 5 above), and extend $\varphi$ to all of $R_{n, 1}$ by first reflecting in the imaginary axis 
and then rotating about the $x_{1}$-axis in $R^{n}$. Then $\varphi$ has the same boundary values as the extremal $u$ in $R_{n, 1}$. Moreover, referring to (8) and (9) we see that $Q_{n} \varphi$ has the same sign in $\mathscr{D}$ as does $-\partial / \partial \beta\left(\left(\left|f^{\prime}\right| / y\right)^{2}\right)$.

Earlier we have shown (cf. (12) in [4]) that

$$
\left(\frac{\left|f^{\prime}\right|}{y}\right)^{2}=\frac{d_{1}^{2}}{s_{1}^{2} c_{1}^{2}}+\frac{k^{4} s^{2}}{c^{2} d^{2}}
$$

where $s, c, d, s_{1}, c_{1}, d_{1}$ have the meaning assigned in (16). But then

$$
\frac{\partial}{\partial s_{1}^{2}}\left(\left(\left|f^{\prime}\right| / y\right)^{2}\right)=\left(-1+2 s_{1}^{2}-k^{\prime 2} s_{1}^{4}\right) /\left(s_{1}^{4} c_{1}^{4}\right)
$$

by $[9$, p. 9] or [10, Formulas 731.01-.03]. Next,

$$
\frac{d}{d s_{1}^{2}}\left(-1+2 s_{1}^{2}-k^{\prime 2} s_{1}^{4}\right)=2\left(1-k^{\prime 2} s_{1}^{2}\right)>0
$$

for $0<\beta<K^{\prime} / 2$, while $\quad-1+2 s_{1}^{2}-k^{\prime 2} s_{1}^{4}=0 \quad$ when $\quad s_{1}^{2}=s n^{2}\left(K^{\prime} / 2, k^{\prime}\right)=1 /(1+k)$ ([9, p. 14], [10, Formula 122.10]). Since also $s_{1}^{2}$ increases with $\beta$ for $0 \leqq \beta \leqq K^{\prime} / 2$, we conclude that $\partial / \partial \beta\left(\left(\left|f^{\prime}\right| / y\right)^{2}\right) \leqq 0$ in $\left.\mathscr{D},{ }^{2}\right)$ so that $Q_{n} \varphi \geqq 0$ in $R_{n, 1}$. Noting that $\varphi=u$ on $\partial R_{n, 1}$ and $Q_{n} u=0$ in $R_{n, 1}$, we may invoke the comparison principle for quasilinear elliptic operators (cf. [16, Theorem 9.2]; see also [8]) to conclude that $\varphi \leqq u$ in $R_{n, 1}$. We now see that if $P \in S^{n-1}$, then

$$
u_{r}(P)=\lim _{a \rightarrow 1^{-}} \frac{1-u(a P)}{1-a} \leqq \lim _{a \rightarrow 1^{-}} \frac{1-\varphi(a P)}{1-a}=\varphi_{r}(P) .
$$

We wish to determine $\varphi_{r}(P)$ for $P \in S^{n-1}$. For this we note first that

$$
\varphi_{r}=\frac{2}{K^{\prime}} \beta_{r}=\frac{2}{K^{\prime}} \operatorname{Im}\left(e^{i \theta} g^{\prime}(z)\right)=\frac{2}{K^{\prime}} \operatorname{Im} \frac{f(\zeta)}{r f^{\prime}(\zeta)},
$$

where $g(z)=\zeta=s n^{-1}(z / \sqrt{k}, k), z=f(\zeta)=r e^{i \theta}$. Using the derivative formula $f^{\prime}(\zeta)=$ $\sqrt{k} c n(\zeta, k) d n(\zeta, k)([9$, p. 9$],[10$, Formula 731.01$])$ and then the Gauss transformation $([9$, p. 72$],[10$, Formula 164.01]) we may reduce (19) to

$$
\varphi_{r}=\frac{2}{(1+k) K^{\prime}} \operatorname{Im} \operatorname{tn}\left((1+k)\left(\alpha+i \frac{K^{\prime}}{2}\right), \frac{2 \sqrt{k}}{1+k}\right)
$$

when $r=1$, where $t n$ denotes $s n / c n$. By [9, p. 38] or [10, Formula 125.01] this is

$$
\varphi_{r}=\frac{2}{(1+k) K^{\prime}} \frac{D S_{1}}{1-S^{2} D_{1}^{2}},
$$

2) The geometric meaning of this inequality is that the hyperbolic density of the rectangle $\left\{(\alpha, \beta):|\alpha|<K, 0<\beta<K^{\prime}\right\}$ is nonincreasing as a function of $\beta$ in the subrectangle $\{(\alpha, \beta)$ : $\left.0<\alpha<K, 0<\beta<K^{\prime} / 2\right\}$. 
where

(20)

$$
S=s n\left((1+k) \alpha, \frac{2 \sqrt{k}}{1+k}\right), \quad D=d n\left((1+k) \alpha, \frac{2 \sqrt{k}}{1+k}\right),
$$

$$
S_{1}=s n\left((1+k) \frac{K^{\prime}}{2}, \frac{1-k}{1+k}\right), \quad D_{1}=d n\left((1+k) \frac{K^{\prime}}{2}, \frac{1-k}{1+k}\right) .
$$

Since $K((1-k) /(1+k))=(1+k) K^{\prime} / 2$ by [9, p. 73] or [10, Formula 164.02] it follows from [9, p. 9] or [10, Formula 122.02] that $S_{1}=1$ and $D_{1}=2 \sqrt{k} /(1+k)$. Thus when $r=1$,

$$
\varphi_{r}=\frac{2}{(1+k) K^{\prime} D}
$$

by $[9$, p. 9] or [10, Formula 121.00], where $D$ has the meaning assigned in (20). But another use of the Gauss transformation reduces (21) to

$$
\varphi_{r}=\frac{2}{(1+k) K^{\prime}} \frac{1+k s^{2}}{1-k s^{2}}
$$

on $S^{n-1}$, where $s$ is as in (16). Combining (22) with (18), we have proved that the second inequality in (15) holds.

To establish the first inequality in (15) we take

$$
\varphi=\varphi(\beta)=\frac{1}{A_{k}} \int_{0}^{\beta}\left(\frac{d n\left(\tau, k^{\prime}\right)}{\operatorname{sn}\left(\tau, k^{\prime}\right) c n\left(\tau, k^{\prime}\right)}\right)^{\frac{n-2}{n-1}} d \tau
$$

in $\mathscr{D}$, and extend $\varphi$ to all of $R_{n, 1}$ by symmetry as before. Then $\varphi=0$ on $\overline{B^{p}(t)}$ and $\varphi=1$ on $S^{n-1}$, and we wish to show that $Q_{n} \varphi \leqq 0$ in $R_{n, 1}$. By Lemma 2 and (17) we see that this reduces to showing that

$$
(n-1) \varphi^{\prime \prime}-\frac{1}{2}(n-2)\left(\frac{d_{1}^{2}}{s_{1}^{2} c_{1}^{2}}+\frac{k^{\prime 4} s^{2}}{c^{2} d^{2}}\right)^{-1}\left(\frac{d_{1}^{2}}{s_{1}^{2} c_{1}^{2}}\right)^{\prime} \varphi^{\prime} \leqq 0
$$

in $\mathscr{D}$, where ' denotes differentiation with respect to $\beta$. But (23) is equivalent to

$$
\left(\frac{d_{1}}{s_{1} c_{1}}\right)^{\prime}\left[\left(\frac{s_{1} c_{1}}{d_{1}}\right)^{\frac{1}{n-1}}-\left(\frac{s_{1} c_{1}}{d_{1}}\right)^{\frac{3-2 n}{n-1}}\left(\frac{d_{1}^{2}}{s_{1}^{2} c_{1}^{2}}+\frac{k^{\prime 4} s^{2}}{c^{2} d^{2}}\right)^{-1}\right] \leqq 0
$$

Since we have already seen that $\left(d_{1} /\left(s_{1} c_{1}\right)\right)^{\prime} \leqq 0$, the problem is reduced to showing that

$$
\left(\frac{s_{1} c_{1}}{d_{1}}\right)^{2}-\left(\frac{d_{1}^{2}}{s_{1}^{2} c_{1}^{2}}+\frac{k^{4} s^{2}}{c^{2} d^{2}}\right)^{-1} \geqq 0 .
$$

But the last inequality is obviously true. Thus $Q_{n} \varphi \leqq 0$ in $R_{n, 1}$. 
Finally, since $\varphi=u$ on $\partial R_{n, 1}$ the comparison principle for $Q_{n}$ says that $\varphi \geqq u$ in $R_{n, 1}$. Then as in (18) we may show that $u_{r} \geqq \varphi_{r}$ on $S^{n-1}$. But on $S^{n-1}$,

$$
\varphi_{r}=\frac{1}{A_{k}}\left(\frac{d_{1}}{s_{1} c_{1}}\right)^{\frac{n-2}{n-1}} \beta_{r}=\frac{1}{A_{k}}(1+k)^{1 /(1-n)} \frac{1+k s^{2}}{1-k s^{2}}
$$

by [9, p. 14] or [10, Formula 122.10] and (22) above. Thus the first inequality in (15) is established.

Lemma 5. If $u$ is extremal for cap $R_{n, n-1}(\sqrt{k})$, then on $S^{n-1}$

$$
\frac{2}{(1+k) K^{\prime}} \frac{1+k s^{2}}{1-k s^{2}} \leqq u_{r} \leqq(1+k)^{\frac{3-2 n}{n-1}} B_{k}^{-1} \frac{1+k s^{2}}{1-k s^{2}},
$$

where

$$
B_{k}=\int_{0}^{K^{\prime} / 2}\left(\frac{s_{1} c_{1}}{d_{1}}\right)^{\frac{n-2}{n-1}} d \beta .
$$

Proof. The function $u$ satisfies the equation (10) in Lemma 3. When $\varphi=\varphi(\beta)$ we see that $Q_{n} \varphi=\operatorname{div}\left(|\nabla \varphi|^{n-2} \nabla \varphi\right)$ has the same sign as does

$$
(n-1) \varphi^{\prime \prime}-\frac{1}{2}(n-2)\left(x /\left|f^{\prime}\right|\right)^{2} \frac{\partial}{\partial \beta}\left(\left(\left|f^{\prime}\right| / x\right)^{2}\right) \varphi^{\prime} .
$$

Now put $\varphi=\varphi(\beta)=2 \beta / K^{\prime}$ in the open first quadrant $\mathscr{D}$ of the unit disk in the $z$-plane, and extend $\varphi$ to all of $R_{n, n-1}$ by reflection across the segment $\{(x, y)$ : $t<x<1, y=0\}$ and then rotation about the $y$-axis in $R^{n}$. Then $\varphi=u$ on the boundary of $R_{n, n-1}$, and (25) is nonpositive in $\mathscr{D}$ if and only if $\partial / \partial \beta\left(\left(\left|f^{\prime}\right| / x\right)^{2}\right) \geqq 0$ there.

By (11) of [4] we may write

$$
\left(\frac{\left|f^{\prime}\right|}{x}\right)^{2}=\frac{1+k^{2} s^{4}}{s^{2}}-\frac{k^{2}+d_{1}^{4}}{d_{1}^{2}}
$$

where $s$ and $d_{1}$ are defined in (16). Then

$$
\frac{\partial}{\partial \beta}\left(\frac{\left|f^{\prime}\right|}{x}\right)^{2}=2 k^{\prime 2} s_{1} c_{1} d_{1}^{-3}\left(d_{1}^{4}-k^{2}\right) \geqq 0
$$

by the derivative formula for $d_{1}([9$, p. 9] or [10, Formula 731.03]) and the fact that $d_{1} \geqq \sqrt{k}$ for $0 \leqq \beta \leqq K^{\prime} / 2$ ([9, p. 14], [10, Formula 122.10]). Thus $Q_{n} \varphi \leqq 0$ in $R_{n, n-1}$, the comparison theorem for $Q_{n}$ shows that $u \leqq \varphi$ in $R_{n, n-1}$, and as in (18) we conclude that $u_{r} \geqq \varphi_{r}$ on $S^{n-1}$. But $\varphi_{r}$ is given by (22) on $S^{n-1}$, and so the first inequality in (24) follows.

To establish the second inequality in (24) we set

$$
\varphi=\varphi(\beta)=\frac{1}{B_{k}} \int_{0}^{\beta}\left(\frac{\operatorname{sn}\left(\tau, k^{\prime}\right) c n\left(\tau, k^{\prime}\right)}{d n\left(\tau, k^{\prime}\right)}\right)^{\frac{n-2}{n-1}} d \tau
$$


in $\mathscr{D}$, and extend $\varphi$ to all of $R_{n, n-1}$ by symmetry as before. Then $\varphi$ has the same boundary values as does $u$, and we wish to show that $Q_{n} \varphi \geqq 0$ in $R_{n, n-1}$. By (25) and (26) this reduces to showing that

$$
\left[\frac{1+k^{2} s^{4}}{s^{2}}-\frac{k^{2}+d_{1}^{4}}{d_{1}^{2}}\right]\left(\frac{s_{1} c_{1}}{d_{1}}\right)^{\prime}+\frac{1}{2}\left(\frac{s_{1} c_{1}}{d_{1}}\right)\left(\frac{k^{2}+d_{1}^{4}}{d_{1}^{2}}\right)^{\prime} \geqq 0,
$$

where' denotes differentiation with respect to $\beta$. By the differentiation formulas $([9$, p. 9] or [10, Formulas 731.01-.03]) and the squared relations ([9, p. 9], [10, Formula 121.00]) for the Jacobian elliptic functions as well as (27) we may show that

and

$$
\frac{d}{d \beta}\left(s_{1} c_{1} / d_{1}\right)=\left(1-2 s_{1}^{2}+k^{\prime 2} s_{1}^{4}\right) / d_{1}^{2}
$$

$$
\begin{aligned}
\frac{d}{d \beta}\left(\left(k^{2}+d_{1}^{4}\right) / d_{1}^{2}\right) & =-2 k^{\prime 4} s_{1} c_{1}\left(1-2 s_{1}^{2}+k^{\prime 2} s_{1}^{4}\right) / d_{1}^{3} \\
& =-2 k^{\prime 4}\left(s_{1} c_{1} / d_{1}\right) \frac{d}{d \beta}\left(s_{1} c_{1} / d_{1}\right) .
\end{aligned}
$$

Since (29) is nonnegative (see the argument after (17) above) it follows from (29) and (30) that (28) is equivalent to

$$
\left(1+k^{2} s^{4}\right) / s^{2}-\left(k^{2}+d_{1}^{4}\right) / d_{1}^{2}-k^{4} s_{1}^{2} c_{1}^{2} / d_{1}^{2} \geqq 0 .
$$

By differentiation it is easy to show that the first term in (31) has a minimum value of $1+k^{2}$. Thus (31) is true in $\mathscr{D}$ if and only if

$$
1+k^{2}-\left(k^{2}+d_{1}^{4}\right) / d_{1}^{2}-k^{\prime 4} s_{1}^{2} c_{1}^{2} / d_{1}^{2} \geqq 0
$$

for $0 \leqq \beta \leqq K^{\prime} / 2$. But it is easy to show by the squared relations for the functions $s_{1}, c_{1}, d_{1}([9$, p. 9], [10, Formula 121.00]) that the left side of (32) is zero. This shows that $Q_{n} \varphi \geqq 0$ in $R_{n, n-1}$, and by the previous reasoning involving the comparison theorem for $Q_{n}$ and an analog of (18) we see that $u_{r} \leqq \varphi_{r}$ on $S^{n-1}$. But

$$
\varphi_{r}=B_{k}^{-1}\left(s_{1} c_{1} / d_{1}\right)^{(n-2) /(n-1)} \beta_{r}
$$

in $\mathscr{D}$, and because $s_{1} c_{1} / d_{1}=1 /(1+k)$ when $\beta=K^{\prime} / 2$, and since $\beta_{r}=(1+k)^{-1}$. $\cdot\left(1+k s^{2}\right) /\left(1-k s^{2}\right)$ when $r=1$ (see (22)), we see that (33) reduces to the upper bound for $u_{r}$ in (24) when $r=1$.

Lemma 6. Let $2 \leqq p \leqq n-2$, where $n \geqq 3$. If $u$ is extremal for cap $R_{n, p}(\sqrt{k})$, then on $S^{n-1}$

$$
(1+k)^{\frac{1}{1-n}} A_{k}^{-1} \frac{1+k s^{2}}{1-k s^{2}} \leqq u_{r} \leqq(1+k)^{\frac{3-2 n}{n-1}} B_{k}^{-1} \frac{1+k s^{2}}{1-k s^{2}},
$$

where $A_{k}$ and $B_{k}$ are defined in Lemmas 4 and 5, respectively. 
Proof. If $u^{(p)}$ and $u^{(p+1)}$ are extremal for cap $R_{n, p}$ and cap $R_{n, p+1}$, respectively, where $1 \leqq p \leqq n-2$, then $Q_{n} u^{(p)}=Q_{n} u^{(p+1)}=0$ in $R_{n, p+1}$, where $Q_{n}$ is the differential operator in (8). Moreover, $u^{(p)}=u^{(p+1)}=1$ on $S^{n-1}$, while $u^{(p)} \geqq u^{(p+1)}=0$ on $\overline{B^{p+1}(\sqrt{k})}$. Hence by the comparison principle for $Q_{n}, u^{(p)} \geqq u^{(p+1)}$ in $R_{n, p+1}$, and the technique used in (18) shows that $u_{r}^{(p)} \leqq u_{r}^{(p+1)}$ on $S^{n-1}$. The bounds in (34) then follow from Lemmas 4 and 5 .

Remark. As an immediate consequence of Theorem 1, (2), and the proof of Lemma 6 , for each $n \geqq 3,1 \leqq p \leqq n-2$, and for each $t \in(0,1)$,

$$
\bmod R_{n, n-1}(t) \leqq \bmod R_{n, p+1}(t) \leqq \bmod R_{n, p}(t) \leqq \bmod R_{n, 1}(t) .
$$

Alternatively, these inequalities are a consequence of the monotoneity of the modulus [14, Lemma 2].

10. An asymptotic limit for the derivative of $\operatorname{cap} R_{n, p}(t)$ as $t$ tends to 0 . We may now combine Theorem 2 with the bounds for $u_{r}$ obtained in the last section to derive bounds for $\mu_{n, p}^{\prime}(t)$ that are asymptotically sharp as $t$ tends to 0 .

Theorem 3. For each $n \geqq 3$ and $1 \leqq p \leqq n-1$, and for $t=\sqrt{k}, 0<k<1$,

$$
\begin{gathered}
A_{k}^{-n}(1+k)^{1 /(1-n)} C_{k} \leqq \mu_{n, 1}^{\prime}(t) \leqq\left(2 / K^{\prime}\right)^{n}(1+k)^{1-n} C_{k}, \\
\left(2 / K^{\prime}\right)^{n}(1+k)^{1-n} C_{k} \leqq \mu_{n, n-1}^{\prime}(t) \leqq B_{k}^{-n}(1+k)^{\left(-2 n^{2}+4 n-1\right) /(n-1)} C_{k}, \\
A_{k}^{-n}(1+k)^{1 /(1-n)} C_{k} \leqq \mu_{n, p}^{\prime}(t) \leqq B_{k}^{-n}(1+k)^{\left(-2 n^{2}+4 n-1\right) /(n-1)} C_{k},
\end{gathered}
$$

where $A_{k}$ and $B_{k}$ are as in Lemmas 4 and 5, respectively, and

$$
C_{k}=2(n-1) \sigma_{n-2} k^{-1 / 2} \int_{0}^{K}\left(\frac{c d}{1-k s^{2}}\right)^{n-2} \frac{1+k s^{2}}{1-k s^{2}} d \alpha .
$$

Proof. By using the derivative formula for $s n$ ([9, p. 9], [10, Formula 731.01]), expressions for the modulus of the elliptic functions ([9, p. 41], [10, Formula 125.01]), the values when $\beta=K^{\prime} / 2$ ([9, p. 14], [10, Formula 122.00]), and the squared relations ([9, p. 9], [10, Formula 121.00]), we may show that

$$
\left|f^{\prime}(\zeta)\right|=(1+k) \frac{1-k s^{2}}{1+k s^{2}}
$$

when $\beta=K^{\prime} / 2 \quad(r=1)$. Likewise we may show that

$$
y=\operatorname{Im} f(\zeta)=\frac{c d}{1+k s^{2}}
$$

there. Thus by Theorem 2, Lemma 4, Fubini's theorem, (38), and (39) (see also [2, 
Section 3]) we have

$$
\begin{aligned}
\mu_{n, 1}^{\prime}(t) \geqq(n-1) k^{-1 / 2} A_{k}^{-n}(1+k)^{n /(1-n)} \int_{S^{n-1}}\left(\frac{1+k s^{2}}{1-k s^{2}}\right)^{n} d \sigma \\
=(n-1) k^{-1 / 2} A_{k}^{-n}(1+k)^{n /(1-n)} 2 \sigma_{n-2} \int_{0}^{K}\left(\frac{1+k s^{2}}{1-k s^{2}}\right)^{n} y^{n-2}\left|f^{\prime}(\zeta)\right| d \alpha \\
=(n-1) k^{-1 / 2} A_{k}^{-n}(1+k)^{1 /(1-n)} 2 \sigma_{n-2} \int_{0}^{K}\left(\frac{c d}{1-k s^{2}}\right)^{n-2} \frac{1+k s^{2}}{1-k s^{2}} d \alpha .
\end{aligned}
$$

This establishes the first half of (35). The second half of (35) follows from the upper bound for $u_{r}$ in (15) by the same reasoning. Likewise (36) follows from Lemma 5. When $2 \leqq p \leqq n-2$, (37) follows from Lemma 6 , the first half of (35), and the second half of (36).

Theorem 4. For $1 \leqq p \leqq n-1, n \geqq 3$,

$$
\lim _{t \rightarrow 0} t\left(\log \frac{1}{t}\right)^{n} \mu_{n, p}^{\prime}(t)=(n-1) \sigma_{n-1} .
$$

Proof. Since $s^{2} \leqq 1$ and $c d \leqq 1$ it is easy to see that $\left(1+k s^{2}\right) /\left(1-k s^{2}\right) \leqq(1+k) /$ $(1-k)$ and $c d /\left(1-k s^{2}\right) \leqq 1 /(1-k)([9$, p. 9], [10, Formula 121.02]). Thus Lebesgue's dominated convergence theorem and the fact that the integrand in $C_{k}$ reduces to $\cos ^{n-2} \alpha$ when $k=0$ ([9, p. 10], [10, Formula 122.08]) allow us to conclude from (37) that

$$
\liminf _{k \rightarrow 0} \sqrt{k} A_{k}^{n} \mu_{n, p}^{\prime}(\sqrt{k}) \geqq 2(n-1) \sigma_{n-2} \int_{0}^{\pi / 2} \cos ^{n-2} \alpha d \alpha .
$$

Since the integral in (40) is $\sigma_{n-1} /\left(2 \sigma_{n-2}\right)([23]$, cf. [4, (3)]) we see that

$$
\liminf _{k \rightarrow 0} \sqrt{k} A_{k}^{n} \mu_{n, p}^{\prime}(\sqrt{k}) \geqq(n-1) \sigma_{n-1}
$$

and

$$
\limsup _{k \rightarrow 0} \sqrt{k} B_{k}^{n} \mu_{n, p}^{\prime}(\sqrt{k}) \leqq(n-1) \sigma_{n-1} .
$$

Finally, by Landen's transformation ([9, p. 72], [10, Formula 163.01]),

$$
d_{1} /\left(s_{1} c_{1}\right)=(1+k) / \operatorname{sn}((1+k) \beta,(1-k) /(1+k)) \geqq 1+k
$$

for $0 \leqq \beta \leqq K^{\prime} / 2$. Since $\lim _{k \rightarrow 0} K^{\prime}=+\infty$ ([9, p. 10], [10, Formula 111.05]), we conclude also that $\lim _{k \rightarrow 0} A_{k}=+\infty$. Then l'Hôpital's rule together with the special values of $s_{1}, c_{1}, d_{1}$ when $\beta=K^{\prime} / 2$ ([9, p. 14], [10, Formula 122.10]) imply that

$$
\lim _{k \rightarrow 0} A_{k} /\left(K^{\prime} / 2\right)=\lim _{k \rightarrow 0}(1+k)^{(n-2) /(n-1)}=1 .
$$

Next, (43) and the fact that the graph of $\operatorname{sn}(\alpha, k)$ is concave down for $0 \leqq \alpha \leqq K$ imply that

$$
s_{1} c_{1} / d_{1} \geqq \beta / K((1-k) /(1+k))
$$


for $0 \leqq \beta \leqq K^{\prime} / 2$. Since $K((1-k) /(1+k))=(1+k) K^{\prime} / 2$ ([9, p. 73], [10, Formula 164.02]), it follows that $\lim _{k \rightarrow 0} B_{k}=+\infty$. Then, as in (44), an application of l'Hôpital's rule shows that

$$
\lim _{k \rightarrow 0} B_{k} /\left(K^{\prime} / 2\right)=\lim _{k \rightarrow 0}(1+k)^{(2-n) /(n-1)}=1 .
$$

Moreover by [9, p. 21] or [10, Formula 112.01]

$$
\lim _{k \rightarrow 0}\left(K^{\prime} / 2\right) / \log (1 / \sqrt{k})=1 .
$$

Recalling that $t=\sqrt{k}$, we see that the limit in the theorem now follows from (41), (42), (44), (46), and (47).

Corollary 1. Let $R_{G, n}(t)$ denote the n-dimensional Grötzsch ring consisting of the open unit ball in $R^{n}$ minus the segment $\left\{P: 0 \leqq x_{1} \leqq t, x_{j}=0,2 \leqq j \leqq n\right\}$. Then

$$
\lim _{t \rightarrow 0} t\left(\log \frac{1}{t}\right)^{n} \frac{d}{d t} \operatorname{cap} R_{G, n}(t)=(n-1) \sigma_{n-1} .
$$

Proof. There exists a conformal mapping of $R_{n, 1}(a)$ onto $R_{G, n}(t)$, where $t=2 a /\left(1+a^{2}\right)$ or $a=\left(1-\left(1-t^{2}\right)^{1 / 2}\right) / t$. Then the corollary follows from Theorem 4 with $p=1$ by a short computation.

\section{Behavior of the derivative of $\operatorname{cap} R_{n, p}(t)$ as $t$ tends to 1 .}

Theorem 5. For each $n \geqq 3$ and $1 \leqq p \leqq n-1$,

$$
\begin{gathered}
\liminf _{t \rightarrow 1}(1-t) \mu_{n, p}^{\prime}(t) \leqq(n-1) \sigma_{n-1}\left(\int_{0}^{\pi / 2}(\sin \beta)^{(2-n) /(n-1)} d \beta\right)^{-n}, \\
\limsup _{t \rightarrow 1}(1-t) \mu_{n, p}^{\prime}(t) \leqq(n-1) \sigma_{n-1}\left(\int_{0}^{\pi / 2}(\sin \beta)^{(n-2) /(n-1)} d \beta\right)^{-n}, \\
\limsup _{t \rightarrow 1}(1-t) \mu_{n, 1}^{\prime}(t) \leqq(2 / \pi)^{n}(n-1) \sigma_{n-1} \leqq \liminf _{t \rightarrow 1}(1-t) \mu_{n, n-1}^{\prime}(t) .
\end{gathered}
$$

Proof. These estimates will follow from Theorem 3 when we have determined the asymptotic behavior of the bounds found there for the derivatives.

First, by $[9$, p. 10] or [10, Formula 111.02],

$$
\lim _{k \rightarrow 1} K^{\prime} / 2=\pi / 4 \text {. }
$$

Then by (43) and (45) we may take the limit under the integral signs in $A_{k}$ and $B_{k}$; by $[9$, p. 10] or [10, Formula 122.08], (48), and a simple change of variable of integration we have

$$
\lim _{k \rightarrow 1} A_{k}=2^{1 /(1-n)} \int_{0}^{\pi / 2}(\sin \beta)^{(2-n) /(n-1)} d \beta
$$

and

$$
\lim _{k \rightarrow 1} B_{k}=2^{(3-2 n) /(n-1)} \int_{0}^{\pi / 2}(\sin \beta)^{(n-2) /(n-1)} d \beta
$$


Next, we determine the behavior of $C_{k}$. Using the Gauss transformation ([9, p. 72], [10, Formula 163.01]), we see that

$$
\int_{0}^{K}\left(\frac{c d}{1-k s^{2}}\right)^{n-2} \frac{1+k s^{2}}{1-k s^{2}} d \alpha=(1+k)^{-1} \int_{0}^{K^{*}} c n^{n-2}\left(\tau, k^{*}\right) d n^{1-n}\left(\tau, k^{*}\right) d \tau
$$

where $\tau=(1+k) \alpha, k^{*}=2 \sqrt{k} /(1+k)$, and $K^{*}=K\left(k^{*}\right)=(1+k) K$. By the change of variable

$$
\xi=\left(k^{*} k^{* \prime} \operatorname{sn}\left(\tau, k^{*}\right) / d n\left(\tau, k^{*}\right)\right)^{2}, \quad d \xi=2\left(k^{*} k^{* \prime}\right)^{2} \operatorname{sn}\left(\tau, k^{*}\right) c n\left(\tau, k^{*}\right) d n^{-3}\left(\tau, k^{*}\right) d \tau
$$

in the last integral, where $k^{* \prime}=(1-k) /(1+k)$, and the squared relations for the elliptic functions ([9, p. 9], [10, Formula 121.00]) we may reduce the second integral in (51) to

$$
\int_{0}^{K^{*}} c n^{n-2}\left(\tau, k^{*}\right) d n^{1-n}\left(\tau, k^{*}\right) d \tau=\left(2 k^{* n-2} k^{* \prime}\right)^{-1} \int_{0}^{k^{* 2}}\left(k^{* 2}-\xi\right)^{(n-3) / 2} \xi^{-1 / 2} d \xi .
$$

By the monotone convergence theorem, the second integral in (52) converges to

$$
\int_{0}^{1}(1-\xi)^{(n-3) / 2} \xi^{-1 / 2} d \xi=2 \int_{0}^{\pi / 2} \cos ^{n-2} \theta d \theta=\sigma_{n-1} / \sigma_{n-2},
$$

where we have made the change of variable $\xi=\sin ^{2} \theta$. Thus

$$
\lim _{k \rightarrow 1}(1-k) C_{k}=(n-1) \sigma_{n-1} .
$$

Finally, by combining Theorem 3 with (48), (49), (50), and (53), we obtain the estimates in Theorem 5 .

Corollary 2. For the n-dimensional Grötzsch ring $R_{G, n}(t)$ the following estimates hold:

$$
\begin{gathered}
\liminf _{t \rightarrow 0}(1-t) \frac{d}{d t} \operatorname{cap} R_{G, n}(t) \geqq \frac{1}{2}(n-1) \sigma_{n-1}\left(\int_{0}^{\pi / 2}(\sin \beta)^{(2-n) /(n-1)} d \beta\right)^{-n}, \\
\limsup _{t \rightarrow 0}(1-t) \frac{d}{d t} \operatorname{cap} R_{G, n}(t) \leqq 2^{n-1} \pi^{-n}(n-1) \sigma_{n-1} .
\end{gathered}
$$

Proof. This follows from Theorem 5 in the same way that Corollary 1 follows from Theorem 4.

12. A surface integral representation for $\mu^{\prime \prime}$. Carrying the method of Section 7 a step further we are able to prove the formula

$$
\mu^{\prime \prime}=(1-n) t^{-2} \int_{S^{n-1}} u_{r}^{n} d \sigma-n(n-1) t^{-2} \int_{S^{n-1}} u_{r}^{n-1} v_{r} d \sigma
$$


for the second derivative of $\mu=\operatorname{cap} R_{n, p}(t)$ with respect to $t$, where $v$ is the coefficient of $\varepsilon$ in the expansion $u^{*}=u+\varepsilon v+\left(\varepsilon^{2}\right)$ of the extremal function for cap $R^{*}$ (see Section 7). The proof is analogous to the proof of Theorem 2 but includes some additional difficulties.

Since $\mu^{\prime}(0+)=\mu^{\prime}(1-)=+\infty$ by Theorems 4 and 5 and since $\mu^{\prime}$ is continuous, $\mu^{\prime \prime}$ cannot have constant sign. However, the proof of (54) shows that the last term in (54) is nonnegative, and if we appeal to Theorem 2 then we conclude that

$$
t \mu^{\prime \prime}+\mu^{\prime} \geqq 0 \text {. }
$$

\section{References}

[1] Anderson, G. D.: Symmetrization and extremal rings in space. - Ann. Acad. Sci. Fenn. Ser. A I Math. 438, 1969, 1-24.

[2] Anderson, G. D.: Extremal rings in $n$-space for fixed and varying $n$. - Ann. Acad. Sci. Fenn. Ser. A I Math. 575, 1974, 1-21.

[3] Anderson, G. D: Dependence on dimension of a constant related to the Grötzsch ring. - Proc. Amer. Math. Soc. 61, 1976, 77-80.

[4] Anderson, G. D.: Limit theorems and estimates for extremal rings of high dimension. - Romanian-Finnish Seminar on Complex Analysis (Proc., Bucharest, 1976). Lecture Notes in Mathematics 743. Springer-Verlag, Berlin-Heidelberg-New York, 1979, 10-34.

[5] Anderson, G. D., and M. K. Vamanamurthy: Estimates for the asymptotic order of a Grötzsch ring constant. - Tôhoku Math. J. 34, 1982, 133-139.

[6] Arfken, G.: Mathematical methods for physicists. - Academic Press, New York-London, 1966.

[7] BoJARSKI, B., and T. IWANIEC: p-harmonic equation and quasiregular mappings. - Sonderforschungsbereich 72, preprint no. 617, Bonn, 1983.

[8] Bojarski, B., and T. IwANIEC: Analytical foundations of the theory of quasiconformal mappings in $R^{n}$. - Ann. Acad. Sci. Fenn. Ser. A I Math. 8, 1983, 257-324.

[9] Bowman, F.: Introduction to elliptic functions with applications. - Dover Publications, Inc., New York, 1961.

[10] Byrd, P. F., and M. D. Friedman: Handbook of elliptic integrals for engineers and physicists. - Springer-Verlag, Berlin-Göttingen-Heidelberg, 1954.

[11] Caraman, P.: $n$-dimensional quasiconformal (QCf) mappings. - Abacus Press, Tunbridge Wells, Kent, England, 1974.

[12] Chester, C. R.: Techniques in partial differential equations. - McGraw-Hill, New York, 1971.

[137 EnNePer, A.: Elliptische Funktionen, Theorie und Geschichte. - Zweite Auflage. Louis Nebert, Halle a. S., 1890.

[14] Gehring, F. W.: Symmetrization of rings in space. - Trans. Amer. Math. Soc. 101, 1961, 499-519.

[15] Gehring, F. W.: Rings and quasiconformal mappings in space. - Trans. Amer. Math. Soc. 103, 1962, 353-393.

[16] Gehring, F. W.: Extremal length definitions for the conformal capacity of rings in space. Michigan Math. J. 9, 1962, 137-150.

[17] Gilbarg, D., and N. S. Trudinger: Elliptic partial differential equations of second order. Springer-Verlag, Berlin-Heidelberg-New York, 1977.

[18] Morrey, C. B., JR.: On the analyticity of the solutions of analytic non-linear elliptic systems of partial differential equations. Parts I and II. - Amer. J. Math. 80, 1958, 198-237. 
[19] NiRENBERg, L.: Remarks on strongly elliptic partial differential equations. - Comm. Pure Appl. Math. 8, 1955, 649-675.

[20] Pólya, G., and M. Schiffer: Convexity of functionals by transplantation. - J. Analyse Math. 3, 1953/54, 245-346.

[21] Rainville, E. D.: Intermediate course in differential equations. - Wiley, New York, 1957.

[22] Ural'ceva, N. N.: Degenerate quasilinear elliptic systems. - Zap. Naučn. Sem. Leningrad. Otdel. Mat. Inst. Steklov 7, 1968, 184-222 (in Russian).

[23] VÄISÄLÄ, J.: Lectures on $n$-dimensional quasiconformal mappings. - Lecture Notes in Mathematics 229. Springer-Verlag, Berlin-Heidelberg-New York, 1971.

[24] ZIEMER, W. P.: Extremal length and conformal capacity. - Trans. Amer. Math. Soc. 126, 1967, 460-473.

Michigan State University

Department of Mathematics

East Lansing, MI 48824

USA

Received 21 June 1984 